\title{
A Case Against Kruppa's Equations for Camera Self-Calibration
}

\author{
Peter Sturm \\ INRIA Rhône-Alpes \\ 655 Avenue de l'Europe \\ 38330 Montbonnot St Martin, France \\ Peter.Sturm@inrialpes.fr
}

\begin{abstract}
We consider the self-calibration problem for perspective cameras, and especially the classical Kruppa equation approach. It is known that for several common types of camera motion, self-calibration is degenerate, which manifests itself through the existence of ambiguous solutions. In a previous paper, we have studied these critical motion sequences and have revealed their importance for practical applications. Here, we reveal a type of camera motion that is not critical for the generic self-calibration problem, but for which the Kruppa equation approach fails. This is the case if the optical centers of all cameras lie on a sphere and if the optical axes pass through the sphere's center, a very natural situation for 3D object modelling from images. Results of simulated experiments demonstrate the instability of numerical self-calibration algorithms in near-degenerate configurations.

Index Terms - Self-calibration, Calibration, Euclidean reconstruction, Kruppa equations, Critical Motions, Degeneracy, Absolute conic.
\end{abstract}

\section{Introduction}

We consider the self-calibration problem for perspective cameras. By self-calibration, we mean the recovery of a camera's intrinsic parameters by only using information contained in images taken by this camera. Explicitly, no information on camera motion or on the 3D structure of the environment is used.

It has been shown by Maybank and Faugeras that, if the camera's calibration remains fixed over an image sequence, self-calibration is in general possible [7]. This result is based on the so-called Kruppa equations, that link the camera's intrinsic parameters with the epipolar geometry of pairs of views taken by the camera. The epipolar geometry can be estimated from sole image point correspondences, so Kruppa's equations put constraints on the intrinsic parameters and can thus be used for self-calibration. Several practical self-calibration approaches based on Kruppa's equations have subsequently been proposed by Faugeras and students of his $[1,5,6,16]$. For other self-calibration approaches, see for example $[2,4,9,10,15]$.

It is known that several types of camera motion exist, for which self-calibration is a degenerate problem, i.e. there exist ambiguous solutions. In $[12,13]$, we report on a complete study 
of the critical motion sequences. The problem of degeneracy must be taken into account in practical self-calibration since several very common imaging situations are indeed critical.

In $\S 4$, we describe a configuration, that is not critical for generic self-calibration, but for which approaches based on Kruppa's equations fail. Concretely, we show that this is the case if all optical centers lie on a sphere and if the optical axes pass through the sphere's center - a situation that appears frequently in 3D object modelling from photographs or image sequences.

In $\S 2$, we briefly introduce a theory of self-calibration on which the rest of the paper is based. Kruppa's equations are reviewed in $\S 3$. In $\S 5$, we examine the instability of Kruppa equation based approaches for self-calibration in near-degenerate situations, through numerical experiments. In $\S 6$, results are shown that underline the fact that other approaches may perform well even in situations that are exactly degenerate for Kruppa's equations. $\S 7$ discusses some special cases of the degeneracy considered in this paper, whereas $\S 8$ provides a general discussion on why certain types of methods suffer from certain types of degeneracies.

\section{Self-Calibration and Euclidean Reconstruction}

We consider camera (self-) calibration as an intermediate step to the recovery of metric 3D structure, also called Euclidean reconstruction. In geometrical terms, obtaining a Euclidean reconstruction is equivalent to determining the position of the absolute conic $\Omega_{\infty}$ [11]. The calibration of a camera's intrinsic parameters is equivalent to the determination of the absolute conic's projection. The absolute conic is characterized as being the only conic in 3-space that is invariant to Euclidean transformations. A consequence of this is that even under arbitrary camera displacements, the projection of $\Omega_{\infty}$ remains fixed, if the camera's calibration does not change during the displacement. This property gives us a constraint for the determination of the absolute conic and its projection, and thus for Euclidean reconstruction and self-calibration.

Euclidean reconstruction or self-calibration can thus be formulated as the determination of the unique conic in 3-space, whose projections are identical in all views of a given image sequence [13]. In this paper, we consider perspective projection as camera model, whose intrinsic parameters are described in the next section. Most practical self-calibration approaches start with a global projective reconstruction and try to identify the absolute conic in 3D; approaches based on Kruppa's equations rely on the epipolar geometry of pairs of views, trying to identify the image of the absolute conic in 2D using local information.

\section{Kruppa's Equations}

Kruppa's equations can be considered as an epipolar matching constraint for the projections of quadrics or conics. Consider figure 1, where the case of a quadric's projection in two views is illustrated. Two epipolar planes are tangent to the quadric, and the induced epipolar lines in the images are thus tangent to the conics obtained by projection of the quadric. Hence, an epipolar line that is tangent to an image conic corresponds to an epipolar line that is tangent to the conic in the other image. The same kind of epipolar constraint is valid if the conics in the images are obtained by projection of a conic in 3-space, instead of a quadric.

If we consider the projections of the absolute conic, we obtain a special case of this conic matching constraint, since the image conics are identical when the images are taken by a 

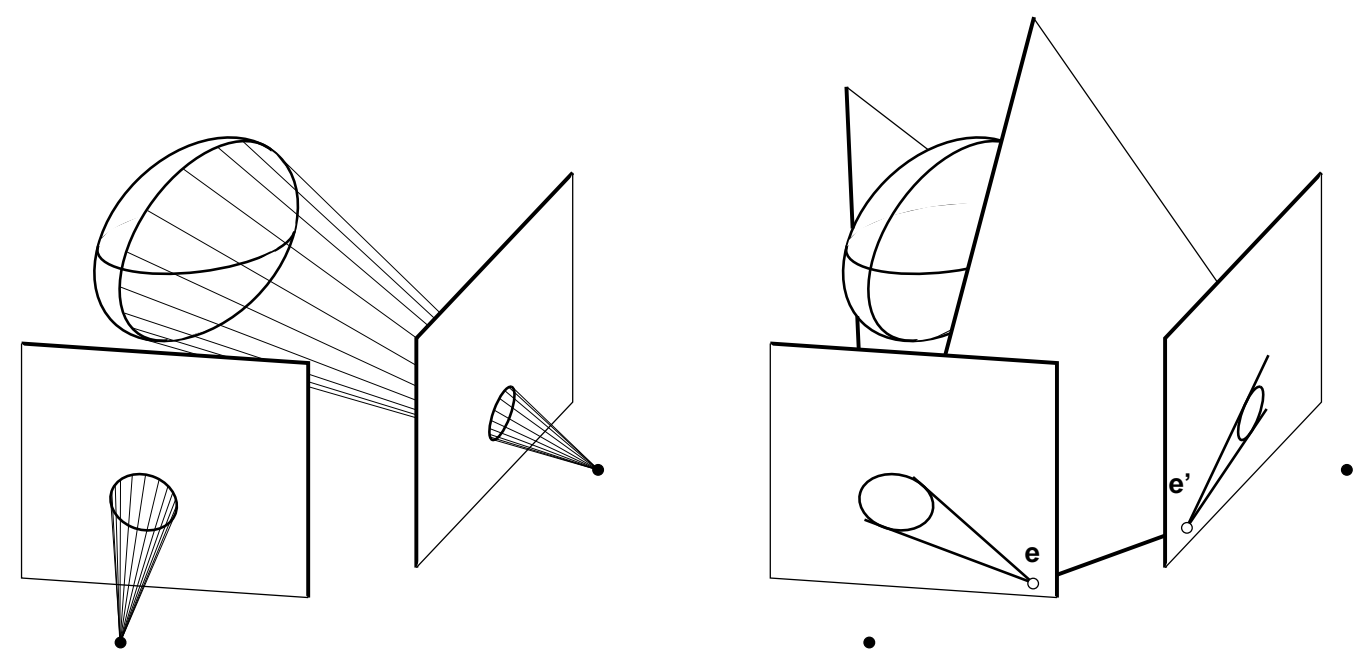

Figure 1: Left: the image of a quadric is the projection of its silhouette, as seen from the optical center of the camera. Right: two epipolar planes are tangent to the quadric; the associated epipolar lines are tangent to the images of the quadric.

camera with fixed intrinsic parameters (cf. §2). Let $\omega$ be the projection of the absolute conic. The matching constraint can be expressed in the following form [16]:

$$
\mathrm{F} \omega^{-1} \mathrm{~F}^{\mathrm{T}} \sim\left[\mathbf{e}^{\prime}\right]_{\times} \omega^{-1}\left[\mathbf{e}^{\prime}\right]_{\times}
$$

where $F$ is the fundamental matrix of the two views, $\mathbf{e}^{\prime}$ is the second epipole (the first epipole $\mathrm{e}$ is not used in this formula), $\sim$ means equality up to scale (we work in homogeneous coordinates) and $\left[\mathbf{e}^{\prime}\right]_{\times}$is the skew-symmetric matrix associated with the cross-product of $\mathbf{e}^{\prime}$. Equation (1) is one formulation of Kruppa's equations. It links the intrinsic parameters of the camera (represented by the image $\omega$ of the absolute conic) with the epipolar geometry (represented by $\mathrm{F}$ and $\mathrm{e}^{\prime}$ ). Since the epipolar geometry can be estimated from sole image correspondences, Kruppa's equations can be used for self-calibration.

Once the image $\omega$ of the absolute conic has been determined using Kruppa's equations or any other approach, the intrinsic parameters are determined straightforwardly, as described in the following. Let the calibration matrix of the camera be given by:

$$
\mathrm{K}=\left(\begin{array}{ccc}
\tau \alpha & -\tau \alpha \cot \Theta & u_{0} \\
0 & \alpha / \sin \Theta & v_{0} \\
0 & 0 & 1
\end{array}\right)
$$

where $\tau$ is the aspect ratio, $\alpha$ the focal length (in pixels), $\left(u_{0}, v_{0}\right)$ the principal point and $\Theta$ the skew angle between pixel axes. The image of the absolute conic in a view with calibration $\mathrm{K}$ is, independently of the view's extrinsic parameters, given by:

$$
\omega \sim \mathrm{K}^{-\mathrm{T}} \mathrm{K}^{-1}
$$

Hence, once $\omega$ is known, the intrinsic parameters are easily determined by Cholesky decomposing $\omega$, using the property of the calibration matrix being upper triangular. Equivalently, but slightly more conveniently, we may decompose the dual of the absolute conic's image:

$$
\omega^{-1} \sim \mathrm{KK}^{\top} \text {. }
$$




\section{A Degenerate Case For Kruppa's Equations}

In this section, we consider the case where the camera to be self-calibrated moves on a sphere while its optical axis points towards the sphere's center. This type of camera motion is not critical for the generic self-calibration problem, but Kruppa's equations are degenerate, which is demonstrated in the following ${ }^{1}$.

Let $\mathbf{C}$ be the center of the viewing sphere (i.e. the sphere of camera positions). Consider an arbitrary sphere $\Phi$ that is also centered in C. Obviously, since the camera is always pointed towards $\mathbf{C}$, the sphere $\Phi$ is perceived in the same way in all views, i.e. its projections are identical. Let $\phi$ be the conic representing the sphere's projections. The fact that $\phi$ is the identical projection of a quadric into all views means nothing else than that $\phi$ satisfies Kruppa's equations for each pair of views. Hence, $\phi$ gives us a mathematically valid, but wrong, solution for the self-calibration problem. We did not constrain the radius of sphere $\Phi$, which means that there is a whole family of ambiguous solutions for self-calibration. Note that this degeneracy is independent of scene structure: the sphere $\Phi$ does not have to exist in the real world - it is a purely algebraic object, like the absolute conic.

In the following, we examine the nature of the ambiguous solutions, i.e. which intrinsic parameters are affected in which way. Let the camera's distance from $\mathbf{C}$ be $d$ and denote spheres centered in $\mathbf{C}$ and with (possibly imaginary) radius $r$ by $\Phi_{r}$. It is easy to verify that the dual of the image of $\Phi_{r}$ is identical in all views and is given by the matrix $\phi_{r}^{-1}$ :

$$
\phi_{r}^{-1} \sim \mathrm{K}\left(\begin{array}{ccc}
\frac{r^{2}}{r^{2}-d^{2}} & 0 & 0 \\
0 & \frac{r^{2}}{r^{2}-d^{2}} & 0 \\
0 & 0 & 1
\end{array}\right) \mathrm{K}^{\top}
$$

Since $\phi_{r}$ is satisfying Kruppa's equations (1), we may try to extract intrinsic parameters from it, as described in $\S 3$ for the true image of the absolute conic. By Cholesky decomposing $\phi_{r}^{-1}$, we obtain an upper triangular calibration matrix $\mathrm{K}_{r}$ satisfying $\phi_{r}^{-1} \sim \mathrm{K}_{r} \mathrm{~K}_{r}{ }^{\top}$. From equations (2) and (3), it follows that $\mathrm{K}_{r}$ is given by:

$$
\mathrm{K}_{r}=\mathrm{K}\left(\begin{array}{ccc}
\frac{r}{\sqrt{r^{2}-d^{2}}} & 0 & 0 \\
0 & \frac{r}{\sqrt{r^{2}-d^{2}}} & 0 \\
0 & 0 & 1
\end{array}\right) .
$$

With regard to the following decomposition of $\mathrm{K}$, separating the focal length $\alpha$ from the other intrinsic parameters:

$$
\mathbf{K}=\left(\begin{array}{ccc}
\tau & -\tau \cot \Theta & u_{0} \\
0 & 1 / \sin \Theta & v_{0} \\
0 & 0 & 1
\end{array}\right)\left(\begin{array}{ccc}
\alpha & 0 & 0 \\
0 & \alpha & 0 \\
0 & 0 & 1
\end{array}\right)
$$

the ambiguous solution for the calibration matrix, due to $\phi_{r}$, is given by:

$$
\mathrm{K}_{r}=\left(\begin{array}{ccc}
\tau & -\tau \cot \Theta & u_{0} \\
0 & 1 / \sin \Theta & v_{0} \\
0 & 0 & 1
\end{array}\right)\left(\begin{array}{ccc}
\frac{r}{\sqrt{r^{2}-d^{2}}} \alpha & 0 & 0 \\
0 & \frac{r}{\sqrt{r^{2}-d^{2}}} \alpha & 0 \\
0 & 0 & 1
\end{array}\right)
$$

\footnotetext{
${ }^{1}$ Triggs already pointed out that this configuration is degenerate for the quasi-linear approach proposed in [15]. Relationships between degeneracies for different types of self-calibration approaches are discussed in $\S 8$.
} 
We observe that the intrinsic parameters given by $\mathrm{K}_{r}$ are identical with the true parameters in $\mathrm{K}$, with the exception of the focal length, which is given by:

$$
\alpha_{r}=\frac{r}{\sqrt{r^{2}-d^{2}}} \alpha
$$

This result has two implications: first, the focal length is usually the intrinsic parameter one is most interested in when self-calibrating (the aspect ratio being constant and often precisely known, the principal point being close to the image center) and it is embarassing that exactly this parameter is obstructed. Second, even prior knowledge of the other intrinsic parameters (aspect ratio, principal point, skew angle) does not help in resolving the ambiguity in focal length estimation!

We now have a closer look at the factor $s=\frac{r}{\sqrt{r^{2}-d^{2}}}$ in equation (4). Depending on the radius $r$ of the sphere $\Phi_{r}$, this factor takes imaginary or real values. The focal length being a real number, we are interested in the cases when $s$ is real. It is easy to check that this is valid exactly in the following two cases:

- $r$ is real and larger or equal than $d$, i.e. $\Phi_{r}$ is a real sphere of equal or larger radius than the viewing sphere. In this case, we have $s \in[1, \infty]$.

- $r$ is a multiple of $I=\sqrt{-1}$, i.e. $\Phi_{r}$ is a sphere of imaginary points only. In this case, we have $s \in(0,1]$.

In conclusion, all non-zero real values are mathematically valid solutions for the ambiguous focal length $\alpha_{r}$.

The reason of Kruppa's equations having ambiguous solutions in a configuration that is not critical for the generic self-calibration problem, may be resumed by the following phrase. Namely, Kruppa's equations are constraints on the image of the absolute conic, but they do not enforce the planarity of the absolute conic in 3D, which is exactly why, in our case, the projections of spheres are admitted as solutions. This issue is discussed in more detail in $\S 8$.

The degeneracy of Kruppa's equations we revealed in this section has been observed in experiments by Zeller and Faugeras [16]: in their self-calibration approach, a global optimization stage is initialized by a robust fit of estimates of the aspect ratio and focal length, obtained from pairs of views and with the principal point being supposed known. The two-view results of the focal length were reported to be extremely unstable, whereas the aspect ratio is estimated reliably. Zeller and Faugeras were not aware that the reason for this seems to be the numerical instability caused by the camera configuration used for their experiments, which is close to the configuration dealt with in this paper. However, during the global optimization stage, when all views are taken into account simultaneously, the instability seems to be reduced enough to obtain good results, which is possible since the camera configuration is only near to, but not exactly, degenerate.

\section{Instability for Near-Degenerate Camera Configurations}

In the following, we report on numerical simulations that have been designed to reveal the instability caused by near-degenerate camera configurations. It is not intended to give a complete quantitative analysis of the problem, but to demonstrate the effect of near-degeneracy on numerical algorithms. 
The basic simulated camera setup is as follows. The scene consists of $503 \mathrm{D}$ points that are randomly chosen in a sphere of radius 100, centered in the origin. We place the camera at arbitrary positions on the sphere of radius 200 , that is also centered in the origin. The camera's calibration is fixed to:

$$
\mathrm{K}=\left(\begin{array}{ccc}
1000 & 0 & 256 \\
0 & 1000 & 256 \\
0 & 0 & 1
\end{array}\right)
$$

and the camera is rotated such that it focuses the origin (the viewing sphere's center).

The following variations and perturbations are applied in various combinations:

- The camera's orientation is changed such that the camera focuses a randomly chosen point within a given distance from the origin (a different point for each view). This distance will be referred to as "optical axis offset" and varies between 0 and 10 .

- The camera is translated off the viewing sphere, toward the origin. Its distance from the origin is reduced from 200 to 180 . The number of translated cameras will be referred to as "number of views off viewing sphere" in the following.

- Gaussian noise of standard deviation 1 pixel is added to the coordinates of the image points.

The first two actions move the configuration away from being critical for Kruppa's equations.

The estimation of the intrinsic parameters is carried out as follows. First, fundamental matrices between pairs of views are estimated by a quasi-linear method [3]. The intrinsic parameters are estimated by a Levenberg-Marquardt type optimization scheme, minimizing a criterion based on Kruppa's equations. The intrinsic parameters are initialized with their true values. Thus, large errors in the estimated parameters indicate large instabilities caused by near-degenerate configurations.

For each setup, we carried out 20 different experiments. In the following graphs, median relative errors for the focal length, the aspect ratio and the coordinates of the principal point are shown. For all of the results shown, eight views have been used for self-calibration.

Figure 2 shows the situation when all cameras are placed exactly on the viewing sphere, but their optical axes are rotated away from the center by various amounts. As the theory in the previous section suggests, the aspect ratio and the principal point are estimated quite reliably even in the exactly degenerate situation when there is no optical axis offset. The error on the focal length however, is, as expected, very large for the degenerate case (note the different scales in the left and right parts of figure 2), and only reaches the same level as for the other parameters, when the optical axis offset becomes significant.

Figure 3 shows the situation when between zero and four of the eight views are translated away from the viewing sphere, but there is no optical axis offset. Again, the aspect ratio and the principal point are estimated with less than 5\% of error (graph not shown). As for the focal length estimation, it is interesting to note that even with only one view taken from a position not on the viewing sphere, the error decreases dramatically, but half of the views have to be translated in order to come close to the $5 \%$ error level.

Finally, figure 4 shows the case of both optical axis offset and views translated away from the viewing sphere. The optical axis offset is such that the cameras focus points within 5 units distance of the viewing sphere's center. It is worth noting that the focal length error drops to less than the half when half of the views are translated off the viewing sphere. 

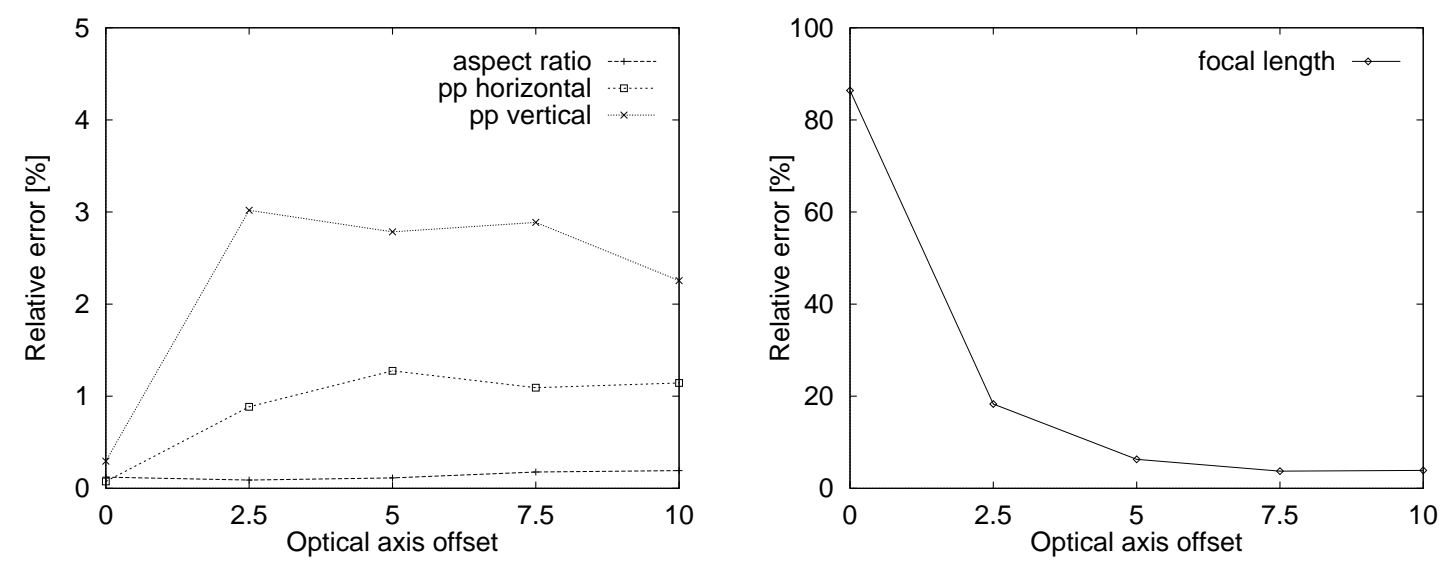

Figure 2: The camera is placed exactly on the viewing sphere, but the optical axis is rotated away from the sphere's center by various amounts. The labels "pp horizontal" and "pp vertical" refer to the principal point coordinates $u_{0}$ and $v_{0}$. Note the different scales in the graphs.

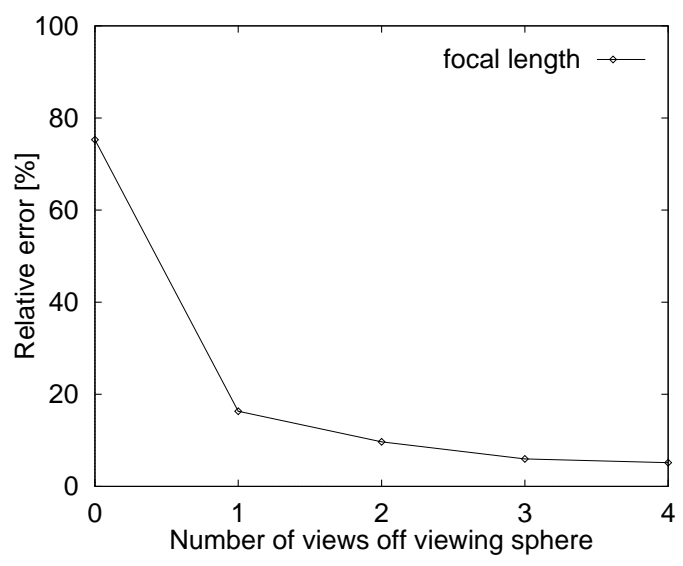

Figure 3: Between 0 and 4 of the 8 views are translated away from the viewing sphere. There is no optical axis offset.

\section{Solving the Present Case}

As we mentioned, the camera configuration discussed in this paper is not inherently degenerate for self-calibration, but for approaches based on Kruppa's equations (and others, cf. §8). Non linear methods which include the planarity constraint for the absolute conic, e.g. [2, 4, 9, 15], will in general succeed in self-calibration (cf. also §8). To demonstrate this, we designed a simple method to resolve the ambiguity introduced by Kruppa's equations. Since the other intrinsic parameters beside the focal length are estimated well by a Kruppa equation approach, we adopt these and, in a second step, apply a method for estimating the focal length only. We want to use the same input as for Kruppa's equations, i.e. fundamental matrices for pairs of views. An alternative is the method proposed in [10], which is based on a global projective reconstruction.

In general, the focal length can be computed from a single pair of views, given the epipolar geometry. Our camera configuration, however, is degenerate for this problem [8]. For triplets of views, focal length estimation is no longer degenerate in general [14]. For each triplet, it 


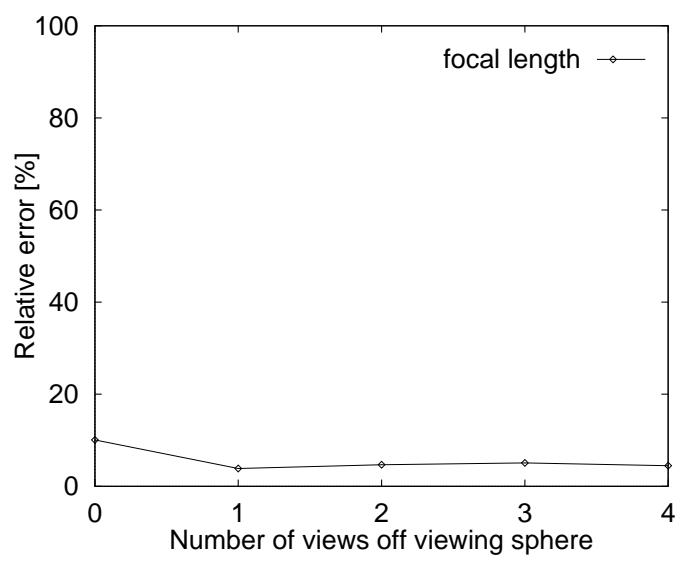

Figure 4: Between 0 and 4 of the 8 views are translated away from the viewing sphere. The optical axes are offset.

is possible to obtain 12 equations of degree 4 in the focal length, with coefficients depending on the 3 fundamental matrices. These equations can be solved individually and their solutions combined in a robust manner to provide an estimate for the focal length. Details of this method are omitted due to lack of space, please contact the author for further information.

Figure 5 shows the relative errors, with respect to the amount of Gaussian noise added to the image points, for the aspect ratio and the principal point (estimated using Kruppa's equations), and the focal length (estimated subsequently). The low error for the focal length confirms that our camera configuration is not inherently critical for self-calibration.

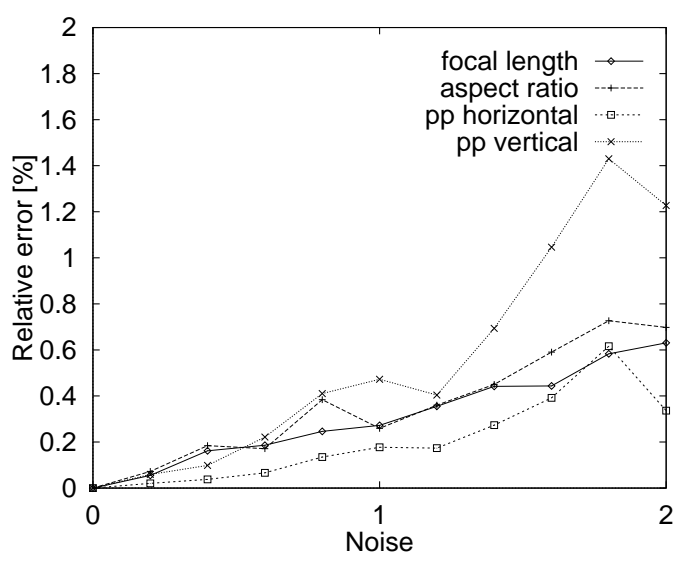

Figure 5: Relative errors for intrinsic parameters with respect to noise in the image points.

\section{Spurious Solutions}

It can be shown that in general, the ambiguous solutions for the focal length described in $\S 4$ represent the only degeneracies for Kruppa's equations with the considered camera configuration. There are however, special cases where further solutions exist, prohibiting the estimation of other intrinsic parameters as well. Additional ambiguities arise for example if there exist quadrics other than spheres whose projections are identical in all views. As we discuss in $\S 8$, 
it would be very difficult to give an exhaustive list of all additional degeneracies and thus to derive conditions under which certain intrinsic parameters can be estimated for sure. However, it is possible to derive some sufficient conditions under which certain parameters can not be estimated without ambiguity. In the following, we describe such conditions for three special cases of the camera configuration discussed in this paper ${ }^{2}$.

The first case concerns cameras located on a circle and fixating this circle's center. We suppose that the cyclotorsion (rotation about the optical axis) is the same for all the views. This configuration is inherently degenerate for self-calibration, giving rise to a 2-degree-offreedom family of solutions [13]. For Kruppa's equations, the ambiguity has at least 3 degrees of freedom. The following conditions hold for cameras with rectangular pixels (i.e. $\Theta=$ $90^{\circ}$; similar but more complicated conditions can be derived for non rectangular pixels). The coordinates of the principal point can never be estimated both at the same time. If the cameras are not upright (i.e. none of the two pixel axes lies in the plane of motion), then none of the coordinates of the principal point can be estimated. If the cyclotorsion is such that none of the pixel axes forms a $45^{\circ}$ angle with the plane of motion, then the aspect ratio can not be estimated. The focal length can never be estimated since we are in a special case of $\S 4$.

The second special case consists again of a camera moving on a circle, but fixating an arbitrary point on the circle's axis, i.e. the optical axes do not lie in the plane of motion. The third case is en extension of this, considering two such sets of cameras, arranged symetrically (i.e. the locus of camera positions is the union of two "parallel" circles of the same size). The ambiguity conditions here are essentially the same as in the first case, except for the aspect ratio, where the $45^{\circ}$ cyclotorsion constraint has to be adapted appropriately to account for the inclination of the optical axes with respect to the plane(s) of motion.

\section{Levels of Degeneracy}

We briefly explain that self-calibration methods may be divided into at least three groups, suffering from increasing levels of degeneracy, i.e. for which increasingly many critical motions exist. Inherent degeneracies, i.e. degeneracies concerning any method, occur exactly if there is a proper virtual conic (i.e. a conic with no real points) in 3-space, different from the absolute conic, whose projections are identical in all views of an image sequence [13].

Methods that do not enforce the planarity ${ }^{3}$ of the absolute conic, suffer from additional degeneracies: camera configurations for which there is a quadric, whose projections are identical in all views, are degenerate. Kruppa's equations are one example: the epipolar matching constraint they represent can not distinguish between quadrics and conics in 3-space. Another example is the linear method proposed by Triggs [15]: the planarity of the absolute conic is a non linear constraint, thus omitted in the linear approach, causing the degeneracy.

In the following, we explain that there exist even more degeneracies for Kruppa's equations, which do not concern the other self-calibration methods cited in this paper. Suppose that $\omega$ is an ambiguous solution for Kruppa's equations. This means that for each pair of views $i$ and $j$, there exists a quadric $\Phi_{i j}$ which projects to $\omega$ in both views. However, Kruppa's equations do not constrain these quadrics to be the same for any pair of views!

\footnotetext{
${ }^{2}$ Results are just summarized here; for details contact the author.

${ }^{3}$ Planarity of the absolute conic is equivalent to rank-3-ness of the "absolute quadric" used in [15].
} 
The three levels of degeneracies are summarized in table 1. Clearly, from top to bottom, there is more and more room for the existence of degenerate configurations. It seems to be quite difficult to describe all the degeneracies for Kruppa's equations explicitly, which is why in $\S 7$ we only give some sufficiency conditions for the existence of spurious solutions.

\begin{tabular}{|l|l|l|}
\hline Methods & Reason for degeneracy & 2D sketch of the reason for degeneracy \\
\hline Any & $\begin{array}{l}\text { Existence of a single conic (repre- } \\
\text { sented by two points here), different } \\
\text { from the absolute conic, with iden- } \\
\text { tical projections in all views }\end{array}$ \\
\hline $\begin{array}{l}\text { Linear } \\
\text { methods }\end{array}$ & $\begin{array}{l}\text { Existence of a single quadric (repre- } \\
\text { sented by an ellipse here) with iden- } \\
\text { tical projections in all views }\end{array}$ \\
\hline $\begin{array}{l}\text { Kruppa } \\
\text { equations }\end{array}$ & $\begin{array}{l}\text { Existence of one quadric per pair } \\
\text { of views, the projections of which } \\
\text { in the associated pair of views be- } \\
\text { ing identical with those of the other } \\
\text { quadrics in the respective views }\end{array}$ \\
\hline
\end{tabular}

Table 1: Different levels of degeneracy, affecting different types of self-calibration methods. Each method inherits the degeneracies from the levels above it.

\section{Conclusion}

In this paper, we have considered the camera self-calibration problem and the classical practical approach, based on Kruppa's equations. We have revealed that for one of the most natural imaging situations (camera moving on a sphere while focusing the sphere's center) this approach fails, in spite of self-calibration being possible in general. Precisely the focal length can not be estimated, even when the other intrinsic parameters are known.

The occurrence of serious numerical instabilities due to near-degenerate camera configurations has been demonstrated by experiment. However, our results suggest that it should be relatively easy to avoid this problem in practice, either by introducing sufficient variation in the camera placement or by using a method that does not suffer from the degeneracy.

We have shown informally that Kruppa's equations suffer from more degeneracies than other known self-calibration methods, which is a prize paid for using local information (fundamental matrices), as opposed to starting with a global projective reconstruction. In general, this paper contributes to the understanding of how to successfully apply self-calibration, which needs good algorithms but also awareness of degenerate situations. 
Acknowledgements. We thank Bill Triggs for fruitful discussions. This work is partly supported by the EPSRC funded project GR/K89221 (Vector).

\section{References}

[1] O.D. Faugeras, Q.T. Luong, and S.J. Maybank, "Camera Self-Calibration: Theory and Experiments," Proc. European Conf. Computer Vision, Santa Margherita Ligure, pp. 321-334, May 1992.

[2] R.I. Hartley, "Euclidean Reconstruction from Uncalibrated Views," Proc. Workshop on Applications of Invariants in Computer Vision, Azores, pp. 187-202, Oct. 1993.

[3] R.I. Hartley, "In Defence of the 8-Point Algorithm," Proc. Int'l Conf. Computer Vision, Cambridge, MA, pp. 1064-1070, June 1995.

[4] A. Heyden and K. Åström, "Euclidean Reconstruction from Constant Intrinsic Parameters," Proc. Int'l Conf. Pattern Recognition, Vienna, Vol. I, pp. 339-343, Aug. 1996.

[5] Q.T. Luong, Matrice fondamentale et autocalibration en vision par ordinateur. PhD Thesis, Université de Paris-Sud, Orsay, France, 1992.

[6] Q.T. Luong and O.D. Faugeras, "An Optimization Framework for Efficient SelfCalibration and Motion Determination," Proc. Int'l Conf. Pattern Recognition, Jerusalem, pp. 248-252, Oct. 1994.

[7] S.J. Maybank and O.D. Faugeras, "A Theory of Self Calibration of a Moving Camera," Int'l Journal of Computer Vision, Vol. 8, No. 2, pp. 123-151, 1992.

[8] G.N. Newsam, D.Q. Huynh, M.J. Brooks, and H.P. Pan, "Recovering Unknown Focal Lengths in Self-Calibration: An Essentially Linear Algorithm and Degenerate Configurations," Proc. ISPRS-Congress, Vienna, Vol. XXXI, Part B3, pp. 575-580, 1996.

[9] M. Pollefeys and L. Van Gool, "A Stratified Approach to Metric Self-Calibration," Proc. Conf. Computer Vision and Pattern Recognition, Puerto Rico, pp. 407-412, June 1997.

[10] M. Pollefeys, R. Koch, and L. Van Gool, "Self-Calibration and Metric Reconstruction in Spite of Varying and Unknown Internal Camera Parameters," Proc. Int'l Conf. Computer Vision, Bombay, pp. 90-95, Jan. 1998.

[11] J.G. Semple and G.T. Kneebone, Algebraic Projective Geometry. Oxford Science Publication, 1952.

[12] P. Sturm, "Critical Motion Sequences for Monocular Self-Calibration and Uncalibrated Euclidean Reconstruction," Proc. Conf. Computer Vision and Pattern Recognition, Puerto Rico, pp. 1100-1105, June 1997.

[13] P. Sturm, Vision 3D non calibrée: contributions à la reconstruction projective et étude des mouvements critiques pour l'auto-calibrage. PhD Thesis, INPG, Grenoble, France, 1997.

[14] P. Sturm, "Critical Motion Sequences for the Self-Calibration of Cameras and Stereo Systems with Variable Focal Length," Proc. British Machine Vision Conf., Nottingham, Sep. 1999.

[15] B. Triggs, "Autocalibration and the Absolute Quadric," Proc. Conf. Computer Vision and Pattern Recognition, Puerto Rico, pp. 609-614, June 1997.

[16] C. Zeller and O. Faugeras, "Camera Self-Calibration from Video Sequences: the Kruppa Equations Revisited,” Research Report 2793, InRIA, Feb. 1996. 